\title{
EMBEDDING PHENOMENA BASED UPON DECOMPOSITION THEORY: LOCALLY SPHERICAL BUT WILD CODIMENSION ONE SPHERES
}

\author{
ROBERT J. DAVERMAN ${ }^{1}$
}

\begin{abstract}
For $n \geqslant 7$ we describe an $(n-1)$-sphere $\Sigma$ wildly embedded in the $n$-sphere yet every point of $\Sigma$ has arbitrarily small neighborhoods bounded by flat $(n-1)$-spheres, each intersecting $\Sigma$ in an $(n-2)$-sphere. Not only do these examples for large $n$ run counter to what can occur when $n=3$, they also illustrate the sharpness of high-dimensional taming theorems developed by Cannon and Harrold and Seebeck. Furthermore, despite their wildness, they have mapping cylinder neighborhoods, which both run counter to what is possible when $n=3$ and also partially illustrate the sharpness of another high-dimensional taming theorem due to Bryant and Lacher.
\end{abstract}

1. Introduction. We shall say that an $(n-1)$-manifold $\Sigma$ embedded in an $n$-manifold $M$ is locally spherical if each point $p$ of $\Sigma$ has arbitrarily small neighborhoods $U_{p}$ such that, in each case, the frontier of $U_{p}$ is an $(n-1)$-sphere $S_{p}$ and $S_{p} \cap \Sigma$ is connected. This definition coincides precisely with the way the term was used until about 1970. Then, however, J. W. Cannon [5] modified the term somewhat for his own ends, saying that such a manifold $\Sigma$ is locally spherical if each point has small neighborhoods with frontiers $S_{p}$ being $(n-1)$-spheres such that $S_{p}-\Sigma$ is simply connected (but necessarily disconnected). When we refer to the concept used by Cannon, we will speak of $\Sigma$ being locally spherical in the sense of Cannon. Of course, in case $n=3$ the two formulations are equivalent.

Before Cannon eventually resolved it, the question of whether a locally spherical 2-sphere $\Sigma$ in the 3-sphere $S^{3}$ must be flat attracted considerable attention and admitted several partial results. The first pertaining to this subject was by $\mathrm{O}$. G. Harrold [12], who proved that such a sphere $\Sigma$ is flat if, in each case, $S_{p} \cap \Sigma$ is a tame simple closed curve. Later C. E. Burgess [3] showed, among other things, how to dispense with the hypothesized tameness of the curve $S_{p} \cap \Sigma$ in Harrold's result. L. D. Loveland [16] established that $\Sigma$ is flat if each $S_{p}$ is flat (and intersects $\Sigma$ in a continuum), and W. T. Eaton [10] did the same without any flatness hypothesis on $S_{p}$, if $S_{p} \cap \Sigma$ is a continuum that irreducibly separates $S_{p}$. Cannon's characterization

Received by the editors September 21, 1981 and, in revised form, April 1, 1982.

1980 Mathematics Subject Classification. Primary 57N50, 57N45; Secondary 54B15, 57N15, 57M30.

Key words and phrases. Wild embedding, locally flat, codimension one sphere, locally spherical, locally weakly flat, homology cell, upper semicontinuous decomposition.

${ }^{1}$ This material is based upon work supported by the National Science Foundation under Grant No. MCS 81-02741. 
of taming sets for 2-spheres [4] gave an improvement of Loveland's work revealing $\Sigma$ to be flat if each $S_{p} \cap \Sigma$ is a continuum that lies on some flatly embedded 2-sphere in $S^{3}$.

For higher dimensions Harrold and C. L. Seebeck [13] introduced a much more rigid concept, saying that an $(n-1)$-sphere $\Sigma$ in the $n$-sphere $S^{n}$ is locally weakly flat if each point $p$ of $\Sigma$ has arbitrarily small neighborhoods bounded by a flat $(n-1)$-sphere $S_{p}$ such that $S_{p} \cap \Sigma$ is an $(n-2)$-sphere flatly embedded both in $S_{p}$ and in $\Sigma$; they showed that every locally weakly flat $(n-1)$-sphere $\Sigma$ in $S^{n}$ is flat.

The results mentioned above, with the solitary exception of Harrold and Seebeck's in the case $n=4$, were all extended by Cannon's result [5, Theorem 5.1] that an $(n-1)$-sphere in $S^{n}(n \neq 4)$ must be flat if it is locally spherical in the sense of Cannon.

In this paper we set forth examples of an $(n-1)$-sphere $\Sigma$ in $S^{n}(n \geqslant 6)$ that is wildly embedded but locally spherical (in the classical sense). Indeed, each point $p$ of $\Sigma$ has arbitrarily small neighborhoods bounded by a flat $(n-1)$-sphere $S_{p}$ and $S_{p} \cap \Sigma$ is (it turns out) a simply connected ANR; moreover, whenever $n \geqslant 7, S_{p} \cap \Sigma$ is an $(n-2)$-sphere. Such $(n-2)$-spheres cannot be standardly embedded in $S_{p}$, nor even have simply connected complement there, for that would conflict with Cannon's result.

These examples provide hard evidence that in any definition of local sphericality the niceness of the embedding of $S_{p} \cap \Sigma$ in $\Sigma$ is much more conducive to forcing tameness than the particular structure of $S_{p} \cap \Sigma$ itself. In that way they demonstrate the strength of Cannon's result. Although variations to it may be possible, these examples suggest that his hypotheses are relatively minimal. Furthermore, they reveal immediately that high-dimensional versions of Loveland's and Eaton's results fail, and that in the Harrold-Seebeck theorem the hypothesis that $S_{p} \cap \Sigma$ be standardly embedded in $S_{p}$ is necessary.

In another vein, we say that a subset $X$ of $S^{n}$ has manifold mapping cylinder neighborhoods provided that $X$ has a closed neighborhood $V$ such that $V$ is an $n$-manifold and there exists a map $\psi: \partial V \rightarrow X$ for which $V$ is equivalent to the mapping cylinder $Z_{\psi}$ of $\psi$ under a homeomorphism acting as the identity on $X$. The examples given here rather obviously possess manifold mapping cylinder neighborhoods. Wildness of this form cannot occur in low dimensions, for an $(n-1)$-sphere $\Sigma$ in $S^{n}$ having manifold mapping cylinder neighborhoods is flat if $n=3$ [18] or if $n=4$ [15]. Moreover, Bryant and Lacher [2] have shown that an $(n-1)$-sphere $\Sigma$ in $S^{n}(n \geqslant 5)$ having (manifold) mapping cylinder neighborhoods is flat if, in addition, it is free from each component $U$ of $S^{n}-\Sigma$, which means that for $\varepsilon>0$ there is an $\varepsilon$-map of $\Sigma$ into $U$. Consequently, these examples justify the presence of an extra hypothesis, like freeness, besides the mapping cylinder hypothesis in their work; whether freeness itself implies flatness remains an open problem in all dimensions.

2. The crucial decomposition-theoretic result. Profound recent developments concerning decompositions of manifolds undergird the unusually direct, almost innocuously simple constructions of this paper. The following consequence of those 
developments, which is essentially established in $\$ 2$ of [9], functions as the indispensible fact from decomposition theory to be employed. Readers interested in learning more about the developments leading to this fact are invited to consult the Introduction of [9]. To give appropriate credit, we should remark that the fact depends either on the Cell-like Approximation Theorem of R. D. Edwards [11] or on its predecessor due to J. W. Cannon [6].

Theorem M. Suppose $n \geqslant 5$ is an integer, $k \geqslant 2$ is another integer, $H$ is a nonsimply-connected homology $(n-k)$-cell, $X \subset$ Int $H$ is a spine of $H, C$ is a Cantor set tamely embedded in Int $I^{k}$, and $G$ is the decomposition of $H \times I^{k}$ into singletons and the sets $X \times\{c\}$, where $c \in C$. Then $\left(H \times I^{k}\right) / G$ is a contractible n-manifold.

To be explicit, we should explain that by a homology $(n-k)$-cell, we mean a compact $(n-k)$-manifold having trivial integral homology and that by a spine of a manifold $M$ we mean a subset $X$ of $M$ such that $M-X$ is homeomorphic to $(\partial M) \times[0,1)$.

Theorem $\mathrm{M}$ is proved in [9] for the case $k=2$. The general case follows directly by regarding $C$ as origin $\} \times C^{\prime}$ in $I^{k-2} \times I^{2}$, setting $H^{\prime}=H \times I^{k-2}$ and $X^{\prime}=H$ $\times$ origin $\}$, and applying the version for $k=2$ to the primed objects.

3. The wild codimension one sphere. Fix an integer $n \geqslant 6$. Let $H$ denote a nonsimply-connected homology 3-cell and let $X$ be a 2-dimensional PL spine of $H$ (that is, $H$ PL collapses to $X$, or, equivalently, $H$ is a regular neighborhood of $X$ ) in Int $H$. In addition, let $C$ denote a Cantor set tamely embedded in Int $I^{n-4} \times\{0\} \subset$ $I^{n-3}=[-1,1]^{n-3}$.

Define an $n$-manifold $M$ as the double of $H \times I^{n-3}$ (that is, $M$ results from the disjoint union of two copies of $H \times I^{n-3}$ after identifying corresponding points from their boundaries), and define an $(n-1)$-manifold $\Sigma^{\prime}$ in $M$ as the double of $\left(H \times I^{n-4} \times\{0\}\right)$. Finally, let $G$ denote the (upper semicontinuous) decomposition of $M$ consisting of singletons and the sets $X \times\{c\}$, where $c \in C$, in just one of the copies of $H \times I^{n-3}$, and let $\pi: M \rightarrow M / G$ denote the associated decomposition map.

Proposition. The set $\Sigma=\pi\left(\Sigma^{\prime}\right)$ is an $(n-1)$-sphere wildly embedded in $M / G$, which is homeomorphic to $S^{n}$.

Proof. A straightforward computation based upon the Mayer-Vietoris sequence reveals that $M$ and $\Sigma^{\prime}$ have homology groups isomorphic to those of $S^{n}$ and $S^{n-1}$, respectively. The classical Vietoris Mapping Theorem [1] which attests that $\pi$ induces homology isomorphisms, shows that $\pi(M)$ and $\pi\left(\Sigma^{\prime}\right)$ also have the homology of the appropriate dimensional spheres.

Furthermore, both $\pi(M)$ and $\pi(\Sigma)$ are simply connected. For instance, $\pi(M)$ is naturally expressed as the union of two copies of $\pi\left(H \times I^{n-3}\right)$. By Theorem $\mathrm{M}$ one of these copies is simply connected (contractible), and the fundamental group of the other obviously is the image under inclusion of the fundamental group of the 
(common) boundary. Application of the Siefert-Van Kampen Theorem indicates that $\pi_{1}(M / G)$ is trivial. Similarly, $\pi_{1}(\Sigma)$ is trivial.

According to Theorem $\mathrm{M}$, both $M / G$ and $\Sigma$ are manifolds (of dimensions $n$ and $n-1$, respectively). Since they are simply connected homology spheres, Newman's topological version of Smale's proof for the Generalized Poincare Conjecture shows that they are topological spheres [17].

The Cantor set $K=\pi(X \times C)$, where $X \times C$ denotes the subset of $M$ in the "correct" copy of $H \times I^{n-3}$, provides the clue to the wildness of $\Sigma$. If $K$ were tame, it would be defined by $n$-cells in $S^{n} \approx M / G$, and the inverse image under $\pi$ of the boundary of a sufficiently small cell would give rise to a simply connected $(n-1)$ manifold separating $\partial\left(H \times I^{n-3}\right)$ from $X \times\left\{c_{0}\right\}$ (for some $c_{0} \in C$ ) in $H \times I^{n-3}$; however, then one could see how to contract any loop from $\partial\left(H \times I^{n-3}\right)$ in $H \times I^{n-3}$, by deforming it to $X \times\left\{c_{0}\right\}$ and cutting the deformation off on the separating manifold, where all loops can be contracted. This impossibility establishes that $K$ is wild in $S^{n}$ (as well as in $\Sigma$ ). (See also [9, p. 181] for a more formal, alternate argument.) Finally, $\Sigma$ must be wild in $S^{n}$ because, by the classical Klee trick [14], every Cantor set in a locally flat $(n-1)$-manifold in $S^{n}$ is tame.

\section{Local sphericality of the sphere.}

THEOREM 1. For $n \geqslant 6$ there exists an $(n-1)$-sphere $\Sigma$ wildly embedded in $S^{n}$ such that each point $p \in \Sigma$ has arbitrarily small neighborhoods bounded by $(n-1)$-spheres $S_{p}$ tamely embedded in $S^{n}$ and intersecting $\Sigma$ in a connected set. Furthermore, for $n \geqslant 7$ these neighborhoods can be constructed so that $S_{p} \cap \Sigma$ is an $(n-2)$-sphere.

Proof. To see that the $(n-1)$-sphere $\Sigma$ of the Proposition is locally spherical, focus on $p \in \Sigma$ and a neighborhood $U$ of $p$ in $S^{n} \approx M / G$. Since $\Sigma$ obviously is locally flat at each point of $\Sigma-K$, we consider only the case in which $p \in K$. Determine a regular neighborhood $N$ of $X$ in $H$ and an open set $V$ in Int $I^{n-3}$ such that

$$
p \in \pi(\text { Int } N \times V) \subset \pi(N \times V) \subset U .
$$

Next determine an $(n-3)$-cell $B$ in $V$ satisfying

(1) $p \in \pi($ Int $N \times \operatorname{Int} B) \subset \pi(N \times B) \subset U$,

(2) $\partial B$ is locally flatly embedded in Int $I^{n-3}$,

(3) $B \cap\left(I^{n-4} \times\{0\}\right)$ is an $(n-4)$-cell $B^{*}$ tamely embedded in $I^{n-4} \times\{0\}$ and standardly embedded in $B$,

(4) $\partial B^{*} \cap C$ is a Cantor set $C^{*}$ tamely embedded in $\partial B^{*}$, and

(5) each point of $C^{*}$ is a limit point of both $C \cap \operatorname{Int} B^{*}$ and $C \cap\left(\left(I^{n-4} \times\{0\}\right)\right.$ $\left.-B^{*}\right)$.

It should be clear how to find $B$ satisfying Conclusions (1)-(4). To obtain (5) as well, name a Cantor set $Z$ in $(-1,1)$ and then use the tameness of $C$ in $I^{n-4} \times\{0\}$ to define a homeomorphism $h: I^{n-4} \times\{0\} \rightarrow[-1,1] \times[-1,1] \times I^{n-6}$ for which $h(C)=Z \times Z \times$ origin $\}$. One can readily spot small $(n-4)$-cells $B^{*}$ about an arbitrary point of $C$ such that $h\left(C \times \partial B^{*}\right)=Y \times\left\{z_{0}\right\} \times$ origin , where $Y$ is open 
and closed in $Z$ and $z_{0}$ is an inaccessible point of $Z$ (i.e., $z_{0}$ belongs to the closure of no component of $(-1,1)-Z)$, and such that $h\left(\partial B^{*}\right)$ meets $[-1,1] \times\left\{z_{0}\right\} \times I^{n-6}$ in an $(n-5)$-cell. The required $(n-3)$-cell $B$ then can be prescribed by thickening $B^{*}$ slightly in the direction orthogonal to $I^{n-4} \times\{0\}$.

Since the compact $n$-manifold $N \times B$ has trivial homology (and cohomology), $\partial(N \times B)$ has the homology of $S^{n-1}$ (see [20, p. 298]). It follows from (3) and (4) that $S_{p}=\pi(\partial(N \times B))$ is an $(n-1)$-manifold. For reasons very similar to those given to justify the Proposition, $S_{p}$ is an $(n-1)$-sphere.

(2) and the definition of $\pi$ should make transparent the fact that $S_{p}$ is locally flatly embedded at each point of $S_{p}-K$. Using [7 or 8 or 19], one can prove that $S_{p}$ is locally flat everywhere by proving $S^{n}-S_{p}$ to be $1-L C$ at each point $q \in S_{p} \cap K$. Towards that end, consider a small loop $L$ near $q$ in, say, Ext $S_{p}$. Find a small regular neighborhood $N^{*}$ of $X$ in $H$ and a small $(n-3)$-cell $D$ in $I^{n-3}-\left(I^{n-4} \times\right.$ $\{0\}$ ) (this requires (3)) such that $L \subset \pi\left(N^{*} \times D\right)$ and $\pi\left(N^{*} \times D\right)$ lies near $q$. (4) and (5) guarantee that $D$ can be chosen so $C \cap \partial D=\varnothing$ and $C \cap D$ is a Cantor set. Again, Theorem $\mathrm{M}$ applies to show that $\pi\left(N^{*} \times D\right)$ is contractible. With a set like $N^{*} \times D$, in lieu of Theorem $\mathrm{M}$ the key to forcing the simple connectivity of $\pi\left(N^{*} \times D\right)$ is the construction providing a nondegenerate element $X \times\{c\}$ of $G$ in $N^{*} \times D$, for any loop $L^{*}$ in $N^{*} \times D$ is homotopic there to a loop in $X \times\{c\}$ and the image under $\pi$ of that homotopy represents a contraction of $\pi\left(L^{*}\right)$ in $\pi\left(N^{*} \times D\right)$. This observation explains why $L$ is contractible in the small set $\pi\left(N^{*} \times D\right) \subset$ Ext $S_{p}$ and clarifies the important role of (5); it also reinforces the argument given above that $S_{p}$ is simply connected.

Finally, note that $\Sigma \cap S_{p}=\pi\left(\partial\left(N \times B^{*}\right)\right)$. No matter what the dimension, this is a connected set. However, it is more interesting when $n \geqslant 7$, for then $\Sigma \cap S_{p}$ can be seen to be an $(n-2)$-sphere, based upon Theorem M, (4), and the arguments establishing that $S_{p}$ is a sphere.

THEOREM 2. For $n \geqslant 6$, there exists an $(n-1)$-sphere $\Sigma$ wildly embedded in $S^{n}$ but having manifold mapping cylinder neighborhoods.

Proof. The sphere $\Sigma$ coincides with that promised in the Proposition. Its mapping cylinder neighborhood $V$ is the image of the two copies of $H \times I^{n-4} \times[-1 / 2,1 / 2]$ in $M$, and the map generating this neighborhood, on either component of $\partial V$, is "translation" to $\Sigma^{\prime}$ followed by the decomposition map $\pi$.

\section{REFERENCES}

1. E. G. Begle, The Vietoris mapping theorem for bicompact spaces, Ann. of Math. (2) 51 (1950), 534-543.

2. J. L. Bryant and R. C. Lacher, Embeddings with mapping cylinder neighborhoods, Topology 14 (1974), 191-201.

3. C. E. Burgess, Characterizations of tame surfaces in $E^{3}$, Trans. Amer. Math. Soc. 114 (1965), 80-97.

4. J. W. Cannon, Characterizations of taming sets on 2-spheres, Trans. Amer. Math. Soc. 147 (1970), 289-299.

$5 . \ldots, U L C$ properties in neighborhoods of embedded surfaces and curves in $E^{3}$, Canad. J. Math. 25 (1973), 31-37. 
6. Shrinking cell-like decompositions of manifolds. Codimension three, Ann. of Math. (2) 110 (1979), 83-112.

7. A. V. Černavskii, The equivalence of local flatness and local 1-connectedness for $(n-1)$-dimensional manifolds in n-dimensional manifolds, Mat. Sb. 91 (133) (1973), 279-286 = Math. USSR Sb. 20 (1973), 297-304.

8. R. J. Daverman, Locally nice codimension one manifolds are locally flat, Bull. Amer. Math. Soc. 79 (1973), 410-413.

9. E__ Embedding phenomena based upon decomposition theory: wild Cantor sets satisfying strong homogeneity properties, Proc. Amer. Math. Soc. 75 (1979), 177-182.

10. W. T. Eaton, A note about locally spherical spheres, Canad. J. Math. 21 (1969), 1001-1003.

11. R. D. Edwards, The topology of manifolds and cell-like maps, (Proc. Internat. Congr. of Mathematicians, Helsinki, 1978), O. Lehto, ed., Academia Scientarium Fennica, Helsinki, 1980, pp. 111-127.

12. O. G. Harrold, Locally peripherally unknotted surfaces in $E^{3}$, Ann. of Math. (2) 69 (1959), 276-290.

13. O. G. Harrold and C. L. Seebeck III, Locally weakly flat surfaces, Trans. Amer. Math. Soc. 138 (1969), 407-414.

14. V. L. Klee, Some topological properties of convex sets, Trans. Amer. Math. Soc. 78 (1955), 30-45.

15. C. Lacher and A. Wright, Mapping cylinders and 4-manifolds, Topology of Manifolds (J. C. Cantrell and C. H. Edwards, eds.), Markham, Chicago, 1970, pp. 424-427.

16. L. D. Loveland, Tame surfaces and tame subsets of spheres in $E^{3}$, Trans. Amer. Math. Soc. 123 (1966), 355-368.

17. M. H. A. Newman, The engulfing theorem for topological manifolds, Ann. of Math. (2) 84 (1966), 555-571.

18. V. Nicholson, Mapping cylinder neighborhoods, Trans. Amer. Math. Soc. 143 (1969), 259-268.

19. T. M. Price and C. L. Seebeck III, Somewhere locally flat codimension one manifolds are flat, Trans. Amer. Math. Soc. 193 (1974), 111-122.

20. E. H. Spanier, Algebraic topologv, McGraw-Hill, New York, 1966.

Department of Mathematics, University of Tennessee, Knoxville, Tennessee 37916 